\title{
Industrial Robots Trajectories Planning for Flexible Manufacturing Systems
}

\author{
Martin Bogdanovskyi and Josef Černohorský
}

\begin{abstract}
Industrial robots (IR) is a popular and perspective technological equipment of modern, complex automation of production processes, which characterized by high precision, speed and flexibility of technological operations. The coordination of robotic end-effectors (EF) provided by advanced automation control systems working on IR and using various sensors and software. The main tasks of IR control systems, which realized in software, include the design of work areas of technological equipment (TE) interaction, planning and on/off line control of IR effectors movements, configuration and synchronization interaction with the TE, human safety control and so on.

The forming of complex approach to solve all of these tasks is of great interest and should take into account the systematization of information about conditions and the complexity of operations, ways of system control and software realizations. Similar approaches are implemented in popular software for IR, such as KUKA Sim, ABB RobotStudio SprutCAM, Octopus, RoboDK etc. which supports C\# -similar languages. At the same time, the problem of effective effectors motion planning in the implementation for continuous trajectory (welding, milling) and discrete, defined among reference points (manipulation, assembly) is not fully solved and often in the real workspace relies on engineering experience. The creation or revision of typical PTP-moving algorithms can take into account such procedures as minimizing time and energy consumption for IR maneuvering abilities and realize it in a short computing time. This article considers the possible methodology approach to plan the IR trajectories in a complex environment.
\end{abstract}

Index Terms-Robotic, trajectory, planning, optimization, energy consumption, genetic algorithm.

\section{INTRODUCTION}

The planning of the IR program trajectories requires to take into account the geometric, kinematic and dynamic properties of IR construction parts, actuators, the realization of program rules for changing the position and orientation of EF, and optimizing their parameters in the space of generalized coordinates. The general approach to solve the planning task in the common, unified methodological basis related with difficulties caused by various task definition, methods of their solution, and the attracted mathematical models. The purpose of the paper is to present one of methods of automated planning of the IR program trajectories to deal with listed problems and to show the practical experience in trajectories optimization and power consumption. According to proposed method [1], the planning process is divided into several stages,

Manuscript received September 22, 2017; revised October 30, 2017

The authors are with Automation and Computer-Integrated Technologies, Zhytomyr State Technological University, Zhytomyr, Ukraine (e-mail: martin.bogdanovskiy@gmail.com). to reach the tasks of service movements inside the TE work areas and the EF regional movements among TE. The different composition and character of the geometric constraints due to the presence in the IR work area the TE parts and the parts of IR itself, which are determined as obstacles during the movement of the IR manipulation system in time of conjugation of the reference points of the program trajectories, determine the general forbidden zone. The IR regional movements planning stage [1] is characterized by an open type of forbidden zones represented by the convex primitives (parallelograms) which used to determine the metric distances and subsequent correction of the trajectory by the genetic algorithm (GA) [2], [3]. This stage deals with problem of optimization the EF movements by taking into account the IR dynamics. Instead, the stage of service movements planning is characterized by the closed and semi-closed type of forbidden zones, determined by the usual design of the TE work areas, which impose significant restrictions on the possible movements of the $\mathrm{EF}$ when leaving the work areas and make the proposed GA [3] for previous stage ineffective in relation to the required computing time. To realize this stage, the modifications to GA are provided.

\section{The State of Problem}

The trajectory planning involves the preliminary formalization and presentation of the parameters of the trajectories of the IR movement. The most natural representation of the IR states is the space of generalized coordinates, which defines the configurations of the IR to the reference points of the trajectories. The process of EF movement planning is characterized by a direct change in the generalized coordinates in time in order to link the target configuration to the "shortest" (optimal or suboptimal according to the chosen criteria), with the exception of inaccessible states. In the motion planning algorithm KPIECE [5], the sampling of the configuration space is performed using a multi-level grid, followed by an estimate of the coverage by the planner agent. The special tree-like graph with branches which describe the IR configuration states represent displacements from the initial configuration in the configuration space is constructed in such a way as to cover as much space as possible outside the forbidden zones. Each movement is determined by the IR configuration state, the controlling influence and the time period during which the controlling influence acts. The iterative application of random control influence in relation to change the generalized coordinates with the estimation of the configuration state of coordinates and multilevel sampling allows to investigate the 
entire configuration space. The advantage of the algorithm is its relative speed in the forming of a moving tree. Instead, in the case of a small number of forbidden zones, this algorithm has computational redundancy and the path found is not always the "shortest". There are also modifications to this algorithm to increase the speed by constructing trees in the neighboring reference points of the trajectory (Bi-directional KPIECE) and checking the Lazy Bi-directional KPIECE algorithm. Another group of PRM algorithms [6] is based on the random generation of IR configurations set in a limited part of the configuration space. The process of determining the local areas of trajectories contains the stage of constructing the graph and approximating the displacements between adjacent configurations to a certain degree of saturation of the "one to many" ratio and the stage of additional vertices generation of the graph determined by the configurations in the adjacent reference points of the trajectory and the search of the optimal path on the graph for Dexter's algorithm. The disadvantages of this algorithm are the unplanned time for solving the problem, which depends on the successful selection of the initial set of IR configurations and significant computational costs. The modification of the PRM algorithm is the SBL algorithm, which generates a tree-like graph between adjacent reference points of the trajectory and the testing of forbidden zones at the end, which improving the speed of the algorithm's computation. The RRT group of algorithms [7] serves to investigate the free zone of the IR configuration space, the idea of which is consist in generation of a tree-like graph of movement from the initial randomized configuration but limited distance at each iteration of generating new configurations with graph branch selection for which the distance to the target configurations in the neighboring point of the trajectory decreases until a certain approach is reached. The disadvantage of relatively simple implementation of the RRT algorithms is reducing the speed with increasing in the number of forbidden zones on the path to the target configuration. Different features of the application of described algorithms for automated IR trajectories planning gave the purpose to search the alternative approaches.

\section{The Trajectories PlanNING IN TE WORK AREA}

The idea of the EF movement planning of the in the TE work areas is to provide the given position and orientation at the point of interaction, as well as the required composition and parameters of IR movements for the exit from the work areas without collisions. At the first stage, the set of target IR configurations is determined at the point of interaction with TE parts on the given vector $R_{l}(x, y, z, a, b, c)=F\left(\bar{q}_{l}^{*}\right)$, where $x, y, z$-Cartesian coordinates of the position of the center of the EF; $a, b, c$ - angles of rotation around the main rails in the global IR coordinate system; $\bar{q}_{l}{ }^{*}$ - vector of generalized coordinates, which determines the vector in solving the direct problem of kinematics. The inverse problem of kinematics with the use of a numerical method of cyclic coordinate descent is solved for the generation of IR configurations set, defined by the vector $\bar{q}_{l}$ of generalized coordinates. In order to ensure the non-local character of approaching the target configurations, the set of random values of the vector (1) of generalized coordinates are formed. Each of coordinates is limited by the types of the joint.

$$
\bar{q}_{l}^{<0>}=\left.\operatorname{Rand}\left(\bar{q}_{\min }<\bar{q}<\bar{q}_{\max }, p\right)\right|_{l=1, L}
$$

where $p$ is the number of initial random generated IR configurations with normal low of distribution.

By fixing the values of all generalized coordinates except one, starting in the order of decreasing the number of a kinematic pair of joints, and minimizing the functional (2):

$$
\Psi\left(\left(\bar{q}_{l}^{<k>}, F\left(\bar{q}_{l}^{*}\right)\right)=\left[F\left(\bar{q}_{l}^{<k>}\right)-F\left(\bar{q}_{l}^{*}\right)\right]^{2}\right) \rightarrow \min
$$

where $\bar{q}_{l}^{<k>}$ - the current vector of generalized coordinates on the i-th iteration;

$\bar{q}_{l}{ }^{*}$ - the desired vector of generalized coordinates,

there is a gradual approximation to its desired value (3):

$$
q_{i}^{<k+1>}=\min _{q_{l}} \Psi\left(\bar{q}_{l}^{<k>}, F\left(\bar{q}_{l}^{*}\right)\right)
$$

For detection of collisions the author propose to calculate the metric distances among all IR and TE parts on work scene [2]. Taking into account the closed or semi-closed types of TE forbidden zones of service at each step of approaching the target configurations defined at the place of interaction between the IR and the TE parts, there is a check for the possibility of collisions. In this case, for estimating collisions, the value of metric distances $d_{z}^{k}$ [2] is calculated.

In the case of absence of collision: $d_{z}^{k}>S_{m}$, where $S_{m}$ is the minimum allowable distance to any parts of the scene, included in the forbidden zone, the increment to the distance is calculated (4):

$$
\Delta d_{z}^{k}=d_{z}^{k+1}-\left.d_{z}^{k}\right|_{z=\overline{1, Z}}
$$

If the collision is present, there is a change in generalized coordinate by the step value for $i$-th joint, proportional to the increase of the metric distance to the change of the generalized coordinate of the joint, defined in the previous iteration, but in the opposite direction (5):

$$
q_{i}^{<k+1>}=\frac{-d_{z}^{k+1} \cdot \min _{q_{l}} \Psi\left(\bar{q}_{l}^{<k>}, F\left(\bar{q}_{l}^{*}\right)\right)}{d_{z}^{k}}
$$

Re-adjusting of current generalized coordinate value continue until condition (5) is reached, after that the coordinate descent continues. If condition (5) is missed and the limit values of the change of generalized coordinates are reached, a transition to the next generalized coordinate is performed. The iterative process of alignment descent continues until one of the two conditions is satisfied (6): 


$$
\Psi\left(\bar{q}_{l}^{<k>}, F\left(\bar{q}_{l}^{*}\right)\right)<\xi d_{z}^{k} \leq S_{m} \forall z=\overline{1, Z}
$$

where $\xi$ - is the accuracy of positioning of the IR EF. In case, the second condition is reached before the first one, the initial configuration is excluded from consideration. The example of realize the metric distance calculation is shown on fig. 1 . The results of calculation show the minimal distance equal to 20 conventional units and the absence of collision. In case collision presents, the type of collision is shown and parts of equipment in collision too. There are 2 types of collision among IR joints only or among IR and TE parts.

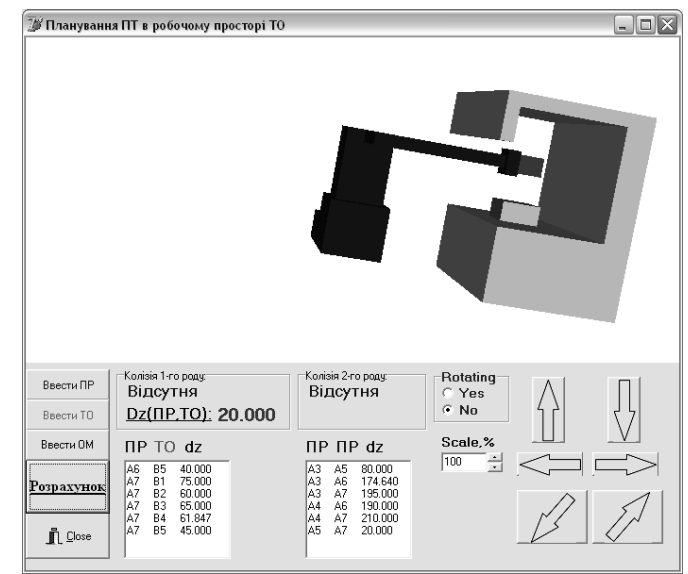

Fig. 1. The calculation of metric distance in semi-closed TE work area.

At the second stage, a set of trajectories which realize non-collision IR removal from TE service area are determined. The determination of the intermediate reference points of the trajectories is performed in the stochastic choice of direction and the value of the step $q_{i}$ in accordance with the normal distribution law. The mathematical expectation of the value of the approximation step varies in proportion to the overall metric distance between IR and TE parts and the mass of the IR joints. The definition of the on-step iteration can be represented as (7):

$$
q_{i}^{<k+l>}=q_{i}^{<k>}+W_{k} \cdot \operatorname{Norm}\left(q_{i}^{<k>}\right)
$$

where $W_{k}$ - the parameter depending on the accumulated weight of the joints in the direction from the EF to the IR basis; $\operatorname{Norm}\left(q_{i}^{<k>}\right)$ - a random scalar, distributed by normal law from the generalized coordinate on the previous iteration.

To implement the stochastic search, the standard deviation of the random scalar $\operatorname{Norm}\left(q_{i}^{<k>}\right)$ decreases in proportion to the incremental value of $\Delta d_{z}^{k}$, and directing the current IR configuration to the target configuration outside TE service area. In case of collision of some IR and TE parts on certain iteration step, the mean square deviation of the scalar $\operatorname{Norm}\left(q_{i}^{<k>}\right)$ to the next step of approximation changes to 1 $\sigma=1$ and the mathematical expectation changes to $m=q_{i}^{k}$. In this way, acceleration of the bypassing of the forbidden zones is realized, especially if they are similar to the closed type. The realization of the second stage begins with the generation of several configurations around the initial at the point of interaction of IR and TE parts in accordance with the normal distribution law with a small dispersion. After that, the definition of the total metric distance is determined. In the case of condition $d_{z}^{k}>S_{m}$ is not reached, the configuration is excluded. Other satisfactory configurations are ranked by and determined by the increment of generalized coordinates by (7). The process is repeated until the increment of the total metric function for all generated configurations at the $k$-step will be positive.

\section{The Thajectories PlanNING AMONG TE Positions}

At the next stage the IR and TE parts are considered as open type forbidden zones. For the purpose of formalization of geometrical, kinematic and dynamic characteristics, the special descriptions of the IR manipulation system and TE parts were used [1]. The main idea of the stage realization is to plan the trajectories at first, taking into account the dynamic IR parameters only in way of using forming of full dynamic math. model in Lagrange form [1] (8):

$$
\dot{x}=A x+b N(u)+f H(q) \ddot{q}+f h(q, \dot{q})
$$

where $x$-vector of the general coordinates direct to actuator; $u$ - actuator control vector; A, b, f - matrix and vector of constructive or electrical parameters of actuators, specified to types. To reach the optimal EF trajectory between the nearest on technological route the minimum "value" of movement cost-function is used (9):

$$
W=\sum_{i=1}^{I} F_{s}\left(x_{s}^{i}, u_{s-1}^{i}\right)=\sum_{i=1}^{I} w_{i}\left(q_{s+1}^{i}-q_{s}^{i}\right)
$$

where $w_{i}=\sum_{l=i}^{I} m_{l} \dot{q}_{l \max }^{2} / 2$. The implementation of function gives similar result to simple PTP algorithms.

The result of the task is presented in form of cubic spline (10):

$$
\begin{aligned}
& h_{n}(t)=\left\lfloor\delta_{n}-v_{f} t_{n}+\frac{a_{f} t_{n}^{2}}{2}\right\rfloor t^{3}+\left(-3 \delta_{n}+3 v_{f} t_{n}-a_{f} t_{n}^{2}\right) t^{2}+ \\
& +\left[3 \delta_{n}-2 v_{f} t_{n}+\frac{a_{f} t_{n}}{2}\right] t+q_{n-1},
\end{aligned}
$$

where $\delta_{n}=q_{f}-q_{n}$ - the increment of a generalized coordinate between indexed points of the trajectory;

$v_{f}, a_{f}, t_{n}$ - velocity, acceleration and time parameters.

After planning the trajectories, the intersection with forbidden zones have to be analyzed. For this propose the discritization of configuration space and metrical function estimation are used [2], [4]. If the collision among the IR and TE parts is detected, the correction of trajectories in the region there it is appear is performed [3]. Such approach of strategy realization for the program trajectories planning gives ability to scale the time computing and functional complexity of the task to forbidden zones configuration. The example of trajectory planning between 4 points relied to TE is shown on Fig. 2. 


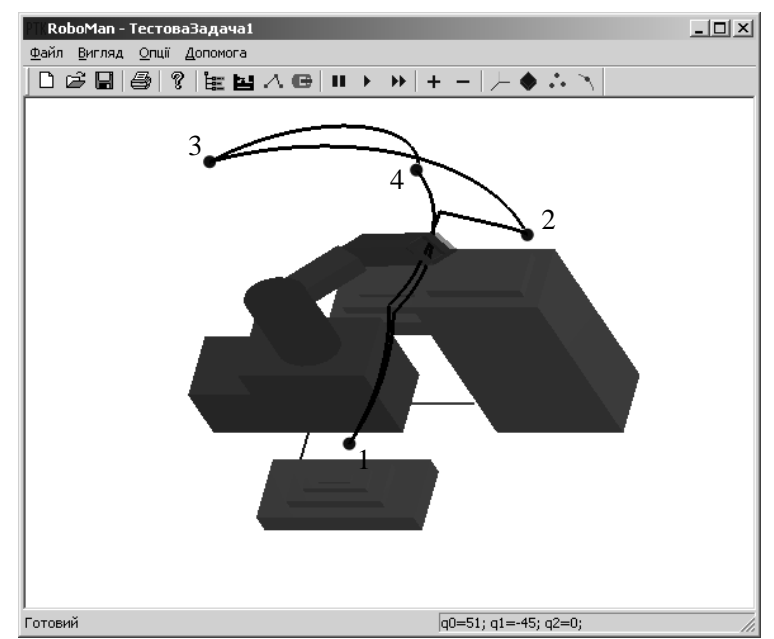

Fig. 2. The EF trajectory planning between TE positions.

The character of trajectory smoothing in case of obstacle avoiding depends on GA parameters and should be optimized by taking into account the real time and energy consumption of IR manipulation system.

\section{TRAJECTORY OPTIMIZATION AND POWER CONSUMPTION}

The other criteria for optimization and even for applying IR are the economic reasons. The cost on robotic side is design and installation, energy and maintenance. The main reason for robotic installation nowadays is reducing the influence of human factor to production and reducing the personal cost ratio in final product. It is very hard to compare different industry and different size of companies. The SME usually outsource the program modification, maintenance, because in overall cost is cheaper then personal cost of skilled personnel. The large factories, like automotive suppliers usually do in the house, or have some extra service contract with robotic application company. The other factor of economical calculations is utilization of robotic system per year which can influence production cost very much. The reducing the cycle time per robotic operation can have the economic benefits even in case of higher power consumption.

The overall robotic power consumption has several components. The energy demanding technology, e.g. welding, seems to have big influence on overall power consumption.

TABLE I: THE RATIO OF THE ENERGY CONSUMPTION OF THE ROBOTIC

\begin{tabular}{l|l|l}
\hline \hline Energy group & Application & $\begin{array}{l}\text { Ratio of energy } \\
\text { consumption }\end{array}$ \\
\hline Robots & Robots - drives & $25.5 \%$ \\
\hline Welding application & $\begin{array}{l}\text { Resistance } \\
\text { spot-welding }\end{array}$ & $7 \%$ \\
\hline Energy-intensive application & Gelation & $9.5 \%$ \\
\hline $\begin{array}{l}\text { Non-energy-intensive } \\
\text { application }\end{array}$ & Gluing & $1.5 \%$ \\
\cline { 2 - 3 } BMS technology & Glue heating & $2 \%$ \\
\hline Stand-by mode & $\begin{array}{l}\text { PLC/SPS, Profinet } \\
\text { components }\end{array}$ & $\begin{array}{l}\text { Stand-by mode of } \\
\text { line }\end{array}$ \\
\hline $\begin{array}{l}\text { Environment (heating,AC, } \\
\text { lighting, pressure air) }\end{array}$ & Factory overall & $50 \%$ \\
\hline Total consumption & \multicolumn{2}{|l}{} \\
\hline \hline
\end{tabular}

The production energy creates more of $50 \%$ of overall factory, the preparation of pressure air is under infrastructure cost, but is used for industrial automation and production.

The robot drives consume almost $26 \%$ of the overall energy, which is a huge number in energy cost in one production year. For that reason even small optimization of trajectory for enhancing the power efficiency can lead to better profit.

If we compare optimized trajectory with common trajectory created by offline programming in offline programming tool, e.g. ABB Robot studio, the power consumption can vary from $80 \%$ less to $20 \%$ more [8]. The simulation of the process and advance control can provide promising results too[9].

The other way, how to reduce the drive cost is in reduction of payload of the robots, e.g. end-effector lightweight design or reducing the mass of technological equipment. Lighter tools could lead to better dynamics and reducing the cycle time. The reducing of payload could lead to using of robot from smaller class and lower investment to robotics.

The usage of energy - saving modes leads to reducing of power consumption during of non-production phase of IR.

The Kuka robots allow Bus Power Off mode and Hibernation mode. The BPO could be activated by digital signal via PLC/SPS. In the BPO mode the robot is still connected to communication network (e.g. Profinet) like active device, but the drives of the robots are disabled. This mode reduces the power consumption even more, that "Stand on brakes".

The other mode with even lower power consumption is Hibernation. This mode leads to disconnecting of robotic controller from industrial network and could lead to error messages in higher control programs. The Wake-On-LAN procedure must be done for controller activation.

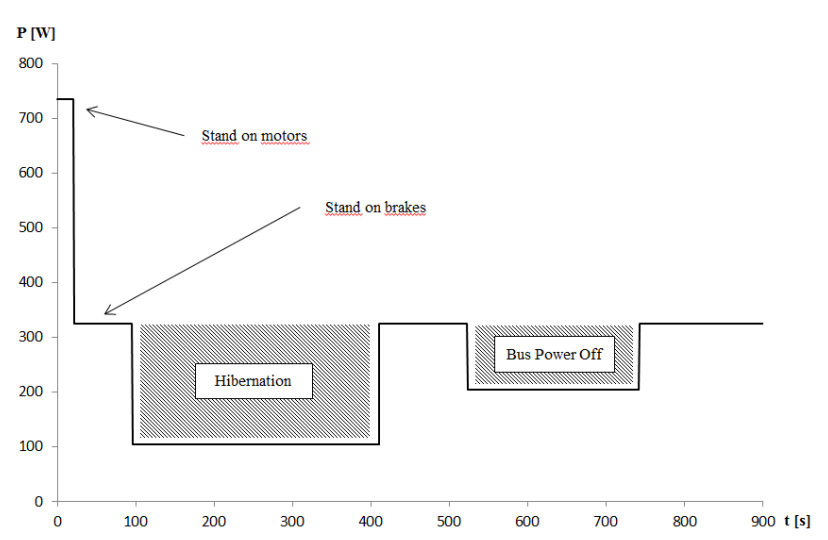

Fig. 3. The energy consumption in energy saving modes [10].

The Fig. 3 present a difference of power consumption on KR210R2700Extra C4 FLR in the home position, the payload of the robot is almost $155 \mathrm{~kg}$. The payload and geometrical position of the robot influence the real power consumption.

TABLE II: THE ENERGY CONSUMPTION IN ENERGY SAVING MODES IN HOME POSITION

\begin{tabular}{l|l|l}
\hline \hline Robot mode & $\begin{array}{l}\text { Robot with } 155 \mathrm{~kg} \\
\text { payload }\end{array}$ & Robot 0kg payload \\
\hline Stand on motors & $735 \mathrm{~W}$ & $420 \mathrm{~W}$ \\
\hline Stand on brakes & $325 \mathrm{~W}$ & $135 \mathrm{~W}$ \\
\hline
\end{tabular}




\begin{tabular}{l|l|l}
$\mathrm{BPO}$ & $205 \mathrm{~W}$ & $115 \mathrm{~W}$ \\
\hline Hibernation & $105 \mathrm{~W}$ & $15 \mathrm{~W}$ \\
\hline \hline
\end{tabular}

The worst mode of parking of the IR is "Stand on motors", where holding torque of the motors is applied and the electromagnetic brakes are activated - released, but the robot is prepared for immediate operation.

\section{CONCLUSION}

The development of algorithms and software based on solving problems of effective trajectory planning of IR effectors by taking into account of the dynamics of movements among the IR trajectories reference points, definition of cost-function that include energy and temporal characteristics and guarantee collisions detection and avoidance is persecuted. For this purpose, the extensive experimental research and examining the situations close to production and creating performance statistics for solving the abovementioned problems are planned to provide. The experiments on software and physical IR models of the forming statistics of energy and natural values estimation through direct and indirect measurement in IR joints links, the power and time consuming estimation in synchronous and asynchronous actuators modes are needed. The trajectory planning and especially the start and end position of the motion influence the power consumption, even in power savings modes. On the other hand, the robot power consumption in the process takes about $25 \%$, the infrastructure cost must be considered too to enhance economic profit.

Acknowledgment: This paper was supported by project FV10099 - Application of the principles "Industry 4.0" in the spinning mills ${ }^{1}$

\section{REFERENCES}

[1] M. Bogdanovskyi and V. Kyrylovich, "Zagadnienia zautomatyzowanego planowania ruchow robotow przemyslowych $\mathrm{w}$ elastycznych systemach montazowych," Technologia i automatyzacja Montazu. Kwartalnik Naukowo-Techniczny, Poland, Warsaw, 1/2009. pp. $18-23$.

[2] M. V. Bogdanovskyi, A. V. Evgenyev, V. A. Kyrylovich, and V. V. Chukhov, "Determination of the metric distance between technological equipment units for the planning of program trajectories of industrial robots of flexible manufacturing cells," Technological Complexes, vol. 1, no. 3, pp. 23-31.

[3] M. V. Bogdanovskyi, and V. A. Kirillovich, "The correction of the program trajectories of industrial robots end-effectors movement during the obstacle avoidance," East European Journal of Advanced Technologies, Scientific Journal, Kharkiv: Technological Center, 2011.

[4] M. V. Bogdanovskyi and V. A. Kirillovich, "The sampling of industrial robots configuration space for the planning of program trajectories," Technological Complexes.

[5] I. A. Sucan, "Kinodynamic motion planning by interior-exterior cell exploration," International Workshop on the Algorithmic Foundations of Robotics, 2008.

[6] L. E. Kavraki, P. Svestka, J. Latombe, and M. H. Overmars, "Probabilistic roadmaps for path planning in high-dimensional configuration spaces," IEEE Transactions on Robotics and Automation, vol. 12, no. 4, pp. 566-580, 1996.

[7] RRT-Connect. Non-optimal planning of robot trajectories [Online]: Available: http://robot-develop.org/archives/3835.

[8] S. Edmonds and R. Lindberg, "Energy optimisation of an industrial robot using iterative dynamic programming," Chalmers University of Technology, Gothenburg, Sweden 2014

[9] K. Kyslan, V. Fedak, F. Durovský, P. Sanjeevikumar, and M. Rodič, "Design and analysis of torque control for load drive with dynamic emulation," in Proc. 2017 International Conference on Optimization of Electrical and Electronic Equipment, pp. 299-304

[10] J. Čejka and J. Černohorský, "Optimization of robotic workplaces," in Proc. the 2016 17th International Carpathian Control Conference, pp. $146-150$

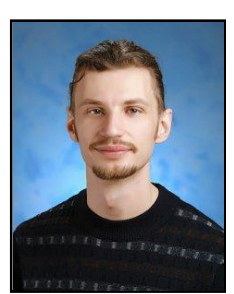

Martin Bogdanovskyi was born in 1980 and he is a assist. prof. Ing. in automation and computer-integrated technologies. Zhytomyr state technological university, Zhytomyr, Ukraine.

His interests are in robotics, mechatronics, industrial automation. 